\title{
Dynamic Robot Formations Using Directional Visual Perception
}

\author{
François Michaud ${ }^{1}$, Dominic Létournea ${ }^{1}$, Matthieu Guilbert ${ }^{1}$, Jean-Marc Valin ${ }^{1}$ \\ ${ }^{1}$ Université de Sherbrooke, Sherbrooke (Québec Canada), laborius@gel.usherb.ca
}

\begin{abstract}
Recent research projects have demonstrated that it is possible to make robots move in formation. The approaches differ by the various assumptions about what can be perceived and communicated by the robots, the strategies used to make the robots move in formation, the ability to deal with obstacle and to switch formations. After suggesting criteria to characterize problems associated with robot formations, this paper presents a distributed approach based on directional visual perception and inter-robot communication. Using a pan camera head, sonar readings and wireless communication, we demonstrate that robots are not only able to move in formation, avoid obstacles and switch formations, but also initialize and determine by themselves their positions in the formation. Validation of our work is done in simulation and with Pioneer 2 robots.
\end{abstract}

\section{Introduction}

Having a group of robots move in formation would be beneficial in many applications in real world environments: exploring an area for surveillance, search and rescue, demining or military missions; manipulating and transporting large objects; surrounding or capturing a prey; convoying, etc. It is then possible for one user to control an entire group of robots, without having to specify explicitly the commands for each one.

While earlier works on robot formations focussed more on theoretical approaches and simulated experiments, the increased capabilities of mobile robots make it now possible to demonstrate their capabilities of moving in formation, avoid obstacles and switch formations. Each approach follows its own set of guidelines regarding the perceptual and communication requirements of the robots, the control strategy and pre-determined knowledge given to the robots, the flexibility of the approach in dealing with obstacles and other constraints of the environment. It is important to clearly identify these guidelines in order to understand the implications of the results demonstrated by the approaches. In this pa- per, we present criteria to characterize problems and approaches for robot formations in order to outline what has been done and what is yet to be accomplished for making robot formations work in real life settings. One capability not yet addressed using robots with limited visibility is the initialization of the formation. We introduce a hybrid control architecture that allows robots to initialize and move in formation, avoid obstacles and switch formations when required, using a distributed approach and by only using directional visual perception. Experimental results obtained in simulation and using Pioneer 2 robots are presented and analyzed, followed by an outline of future work.

\section{Formation Control Problems}

Formations are defined as groups of mobile robots establishing and maintaining some predetermined geometrical shape by controlling the positions and orientations of each individual robots relative to the group, while allowing the group to move as a whole. The coordination problem that has to be solved is local in terms of what each robot has to do, and global at the level of the group. The simplest case involves spatial coordination among robots, and the more complex ones add temporal coordination of robots' trajectories and roles. Most approaches focus on spatial coordination strategies, while temporal coordination would be required for real world use of robot formations.

Different characteristics can be associated with these coordination strategies. While doing our survey of the literature on robot formation control, we noticed that such characteristics are not always clearly outlined. This makes it hard to determine the advantages and limitations of the approaches developed so far. In an attempt to better identify these characteristics, we propose to group them into three categories, as outlined below.

\section{Perceptual characteristics:}

Visibility of other robots. Approaches may consider complete [10] or limited [3, 5] visibility of robots in the group. 
Frame of reference. Robots may use an absolute positioning system to base their decisions [8, 10], or do everything relative to their own reference frame $[3,5]$.

Communication capabilities. Either no communication [10], communication of global information $[3,5,8]$ or local information [5, 8] are possible. Communication allows to compensate for insufficient sensing capabilities at the global level (for coordination of the formation), or at the local level (like the position of the other robots using an absolute frame of reference).

\section{Formation characteristics:}

Types of formation. This relates to the variety of geometrical shapes that an approach can handle. Formation types can be circle, diamond, wedge, line, column, triangle, rectangle, arrowhead, hexagon, tree, lattice (hexagonal, rectangular or triangular), or arbitrary.

Position determination. Three techniques exist: unit-center-referenced, where the average of the $x$ and $y$ positions of all robots is computed and used as a common reference [1]; point-referenced, with each robot determining its position in relation to a single point, which can either be the leading robot (also called leader-referenced [1]) or a "virtual" point (also called virtual structure $[7,8]$ ); and neighborreferenced, in which each robot maintain a position relative to one [5] or up to two [3] robots in proximity.

Neighbor-referenced approaches are required by robots with incomplete perception of the group. Robots that are 'interconnected' to each other can have unidirectional influences (from the preceding robot to its followers) [3, 5], or bidirectional influences (the preceding robot is influenced by its followers, and vice-versa) [12]. The inter-relations between robots can emerge by having robots follow the closest neighbor [10] or be explicitly assigned (using ID numbers for instance $[3,5]$ ).

Structural constraints. Formations can be rigid [8], i.e., they must preserve their shape at all time, or flexible [3].

\section{Control characteristics:}

Decision process. While it is possible to use a centralized [7] approach for formation control, the majority is distributed, making each robot determine what to do autonomously. In that case, the decision process can be either homogeneous if all robots follow the same decision rules, or heterogeneous if robots have distinctions between them to bias their decisions.

Dependence on temporal states. The algorithm can be oblivious if its decisions are determined only from the sensory information observed at that time instant, or non-oblivious if it exploits information from the past [11]. The majority of algorithms are oblivious, except maybe in [5] for which the history of past bearing and distance is used to handle ambiguous situations.

Control strategy. It can be based on control laws (involving for instance input-output feedback linearization [7]), behavior-based [1, 5] or hybrid [4] (i.e., involving a supervisory level and an execution level).

In addition to these characteristics, approaches associated to formation of robots involve different types of coordination problems, listed below from the problem most addressed to the one least studied:

a) Maintain formation shape while moving [1, 3, 4, $7,8,10]$. Criteria like the stability while moving in formation and robustness to robot failure are important [5].

b) Avoid obstacle while occasionally splitting/deforming and then reestablishing the formation $[1,3,4,10]$, or preserving the shape of the formation [5].

c) Change the shape of the formation $[2,5,10]$.

d) Assemble at the starting point and/or establish the formation $[10,11]$.

e) Determination of feasible formations [4, 12].

\section{Formation Control Architect ure Using Directional Visual Perception}

Using the framework of Section 2, we find it easier to outline possible research contributions, what can be exploited and what needs to be refined. In our case, we want to work with robots that only have limited visibility of the environment (using a directional camera and sonar readings), relative frame of reference (no absolute positioning system) and communication capability (for local and global information) to compensate for the limited visibility constraint. Such characteristics are closer to what would prevail in real life settings. We assume that each robot is equipped with a colored cylinder that allow them to perceive their positions and their distances relative to others. However, no information about the orientation of the robots is provided. Similarly to [5], we use neighbor-referenced technique with unidirectional influences, but with a behavior-producing module that gives more flexibility to the formation structure (to tackle coordination problems a) and b)). Also, we do not want to establish formation based on the robot's ID number (as in $[5,3]$ ), but to establish the formation dynamically and address this way coordination problems c) and d). 
Our approach follows the guidelines of a hybrid control architecture that combines local level control of a robot with global level deliberation over the overall states of the group [9]. In this project, only two levels of the architecture are used, i.e, the behavioral level (bottom part) and the recommendation level (highest part). Figure 1 represents the architecture implemented, described in the following subsections.

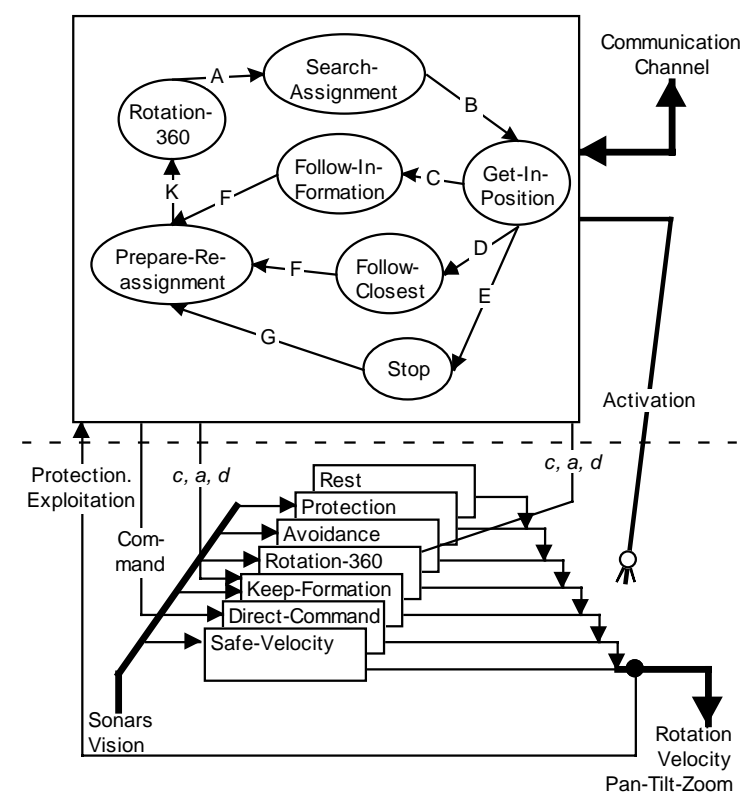

Figure 1: Formation control architecture.

\subsection{Behavioral Level}

Behavior-producing modules allow a robot to respond in particular ways to situations encountered in the environment. These modules all run in parallel and their resulting commands are prioritized to generate the control actions of the robot. The behavioral modules used in our approach do the following: Safe-Velocity makes the robot move forward without colliding with an object; Direct-Command changes the position of the robot according to specific commands; Keep-Formation allows a robot to follow an assigned robot of color $c$, at angle $a$ and at distance $d$; Rotation-360 reinitializes the position of the camera toward the front, and makes the robot rotates slowly in order to localize other robots in its vicinity; Avoidance moves the robot away from obstacles detected (at up to 60-80 $\mathrm{cm}$, depending on $d$ ) based on front sonar readings; Protection monitors the presence of an object very close (at about 20 to $30 \mathrm{~cm}$, also depending on $d$ ) to the robot; Rest makes the robot stop moving.
Using these behavior-producing modules, a robot is capable of following another robot while avoiding obstacles. Since no absolute referencing system is used, robots have to rotate on themselves to detect the presence of other robots nearby. This information is used by the recommendation level to establish the formation. Another distinction of our approach is in the Keep-Formation behavior. The behavior similar to this one in [5] allows a robot to follow another by using laser data for distance, pan the camera at the desired angle and keeps the preceding robot in the center of the field of view of the camera. In our case, Keep-Formation infers distance with other robots using only visual information, and it can adjust the pan angle of the camera according to the position of the colored-blob in the image to keep the preceding robot in its field of view. Proportional controllers are used to keep a robot at the specified angle and distance relative to the assigned robot to follow. The zoom of the camera is not used.

\section{$3.2 \quad$ Recommendation Level}

To address coordination problems c) and d) described in Section 2, coordination at a more global level is required. At initialization, we assume that robots are not positioned appropriately to move as the desired formation. The objective is to have the robots determine a leader for the desired formation, and get assigned to follow a robot (with angle and distance constraints) to establish the formation.

The algorithm we propose is implemented as a simple finite state machine, as shown in the upper part of Figure 1. Behavior-producing modules get activated based on these states. When a desired formation is given to the group, each robot enters into the Rotation-360 state, making them rotate on themselves to perceive nearby robots. The perceived information is shared between robots so that each of them can initialize its Visibility Table. A Visibility Table is a $n$ by $n$ table, $n$ being the number of robots in the group. Each $(i, j)$ entry of the table memorizes the angle and the distance of robot $j$ if seen by robot $i$. Robot $i$ initializes line $i$ of the table with the information it perceives using its camera, and other lines with the information communicated by the other robots. Note that the algorithms does not verify mutual perception of robots: it is possible to have robot 1 see robot 2 , and robot 2 not seeing robot 1 .

Once these tables are initialized for each robot in the group (condition A shown in Figure 1), the robots enter into the Search-Assignment state. The objective is to have robots search for a good assignment of robots to establish the desired formation, based on information gather in the visibility tables. To do 
so, each robot considers itself as being in the leading position for the desired formation, and our approach uses a search algorithm to determine the best possible position assignment for the other robots in the group. The search algorithm evaluates, for all possible assignations, the following cost function that must be minimized:

$$
\sum_{i=1}^{n} \sum_{j=1}^{n f_{i}(f)} \frac{\left(\triangle d_{i, j}\right)^{2}+\left(d_{i, j} \cdot \triangle a_{i}\right)^{2}}{\text { Level }_{i}(f)}
$$

where $n f_{i}(f)$ is the number of robots that must follow robot $i$ in formation $f, \triangle$ represents the difference between the desired and perceived parameter (distance or angle) in the formation. The equation is an approximation of the total square distance between the initial and the desired positions of robots in the formation, which we cannot compute without having an absolute referencing system.

Since robots only perceive others in proximity, it may not be possible to assign robots to follow others that they see, according to the desired formation. By dividing by Level, the cost function allows to favor non-visible assignations at the extremities of a formation. Our algorithm uses a bounded depth-first search and starts finding solutions iteratively from 0 to $n$ non-visible robots in the formation. The search process stops as soon as the best formation is found for a minimum number of non-visible robots. In a particular assignation, if robot $j$ does not see robot $i, d_{i, j}$ is set to a large value, making the search process try a new assignation with a higher number of robots assigned to follow a robot not directly visible. By having each robot search for the best assignation with itself as the formation leader, the group conducts a distributed search for the best assignment possible. The best result obtained by each robot is broadcasted to the others, and the one with the best overall score is elected as the leader of the winning formation.

Once robots have reached a consensus (condition B), they all know which robot to follow (i.e., its color $c$ ), at what angle $a$ and distance $d$. For the GetIn-Position state, robots are allowed to turn to the right heading to see the robot to follow (condition C). Remember that the search algorithm does not preclude the assignation of a robot to follow another that is not seen. In that case, the robot is assigned to follow the closest robot (condition D). Otherwise, if the robot does not see any other robots, it is set to remain inactive and not to interfere with the other robots (condition E). The formation can then get established by letting the robots move autonomously. The formation leader is set to turn away from nearby robots to minimize congestion while establishing the formation.

While establishing or moving in formation, robots may or may not have difficulties following the assigned robots. For instance, robots that are moving too close to each other may generate interference that make it difficult to establish the formation. Represented by condition F in Figure 1, if one robot looses sight of the robot it has to follow, or if it is experiencing difficulties moving (reflected by the prolonged usage of the Protection behavioral module), then it requests a reassignment to be done for the group. For an inactive robot, this means it will be allowed to try to join the group (condition G). The robots then position themselves at a reasonable distance of any obstacle in proximity (using Protection), and stop. Once the group has stop moving (condition K), the Rotation-360 state is repeated to reevaluate a new assignation for the formation. Note that the formation leader is also allowed to request a reassignment if it has trouble moving. To prevent having a robot that is experiencing difficulties be elected as the new leader, such robot (or robots if there is more than one) will then not consider itself as a potential leader and will not participate in the distributed search process explained previously.

Finally, the Communication Channel link represents all information that is communicated to and between robots. It can be commands from an operator, teleoperating the formation leader or requesting the group to move in a new formation. It also includes information shared by the group (i.e., the visibility tables) or requests for reassignment.

\section{Experimental Results}

We conducted simulated and real world experiments using Pioneer 2 robots equipped with pan-tilt-zoom cameras and sonars. Simulation experiments were done using the Player / Stage environment [6]. Two sets of experiments were conducted: experiments to demonstrate the capabilities of the behaviorproducing modules to make robots move in formation and avoid obstacles, and experiments validating the capability of our approach for establishing a formation.

\subsection{Behavior-Producing Module Experi- ments}

To demonstrate the flexibility added by controlling the pan angle of the camera, we ran experiments and compare results with a version of the Keep-formation behavioral module that keeps the pan angle fixed (as done in [5]). As shown in the upper part of Figure 2 , robots with fixed pan angles cannot avoid obstacles in a flexible manner. That explains why in [5] a robot avoiding an obstacle has to communicate the 
rotation it is making to the other robots in order to keep all robots in the appropriate line of sights of the others. This makes the formation rigid with bidirectional influences between neighbors. Our behavioral module adds the possibility of using directional vision for flexible and unidirectional formations. This can be seen in the lower part of Figure 2, for a robot avoiding an obstacle or for making the group moves near a wall in an arrowhead formation. The simulation results were also confirmed using Pioneer 2 robots.

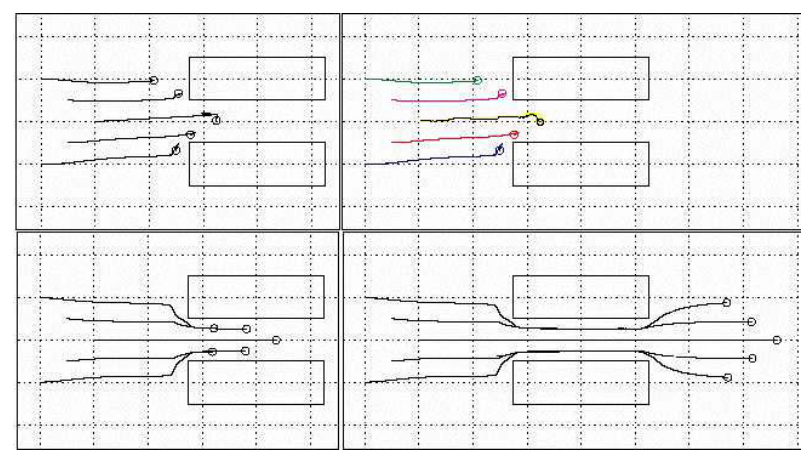

Figure 2: Simulation of robots moving in formation with a fixed pan angle (upper part) and with controlled pan angle (lower part). The experiments are shown moving from left to right.

\subsection{Establishing a Formation}

We conducted a series of experiments, in simulation and with the real robots, to validate the initialization of formations. The results reported in this paper concern arrowhead and column formations. One set of experiments examines how the group can converge to a desired formation, starting from random position. This allows to evaluate the capability of the search algorithm to determine the best possible assignation given the incomplete information perceived by the robots, and the capability of the finite state machine to recover from failure in establishing the formation. As shown at the top of Figure 3, the formation is capable of initializing itself appropriately. Starting from random positions, the group selects the robot in the middle to be the formation leader. The formation then get established in the desired arrowhead configuration.

Autonomous establishing formations becomes an important capability for the group when facing difficult situations. For instance, when a column formation goes in a dead end, our approach allows the group to assign the robot at the end of the column as the new leader, as shown in the middle portion of Figure 3.

We also validated the algorithm for switching formations, changing from arrowhead to column to come

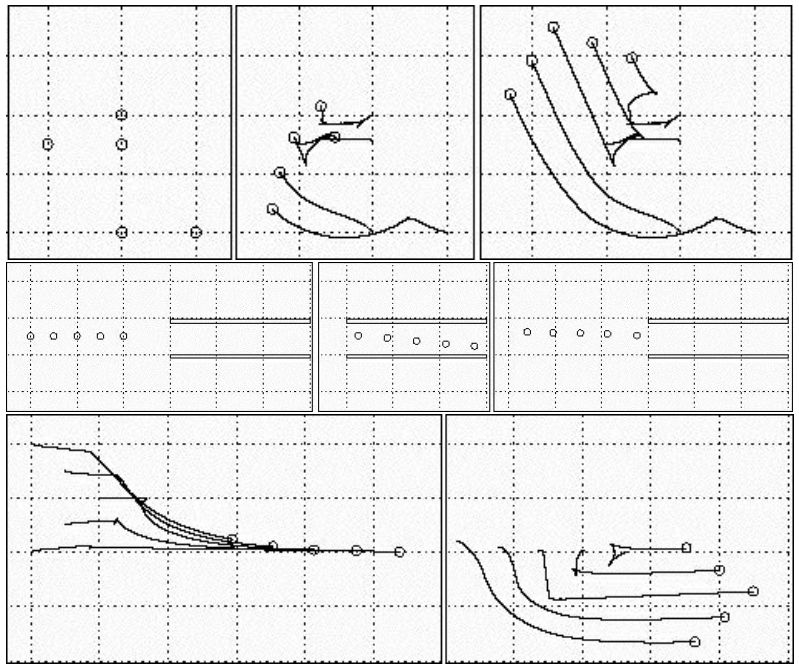

Figure 3: Simulation of: (top) initialization of an arrowhead formation; (middle) reassignation when a column formation goes in a dead end; (bottom) switching from an arrowhead formation to a column, and back again to arrowhead.

back again to arrowhead. The bottom part of Figure 3 illustrates this switch using our approach.

Figure 4 illustrates what happens with a group of robot that starts in a column formation and receives a request for a arrowhead formation. The robots then rotate to localize nearby robots (by rotating on themselves). Once done, the robot in the middle is selected as the new leader, and the arrowhead formation is established ${ }^{1}$.

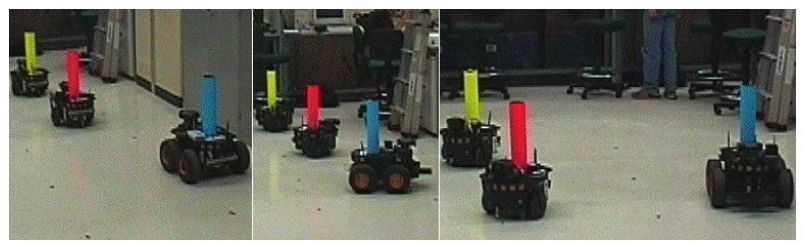

Figure 4: Reassignation of a formation from column to arrowhead.

\section{Discussion}

No approach has yet demonstrated the capability to address all of the coordination problems presented in Section 2. For problems a), b) and c) about formation maintenance, obstacle avoidance and formation switching, the work closest to ours is done by Freslund and Mataric [5]. Using similar perceptual capabilities (but no laser), we have demonstrated that

\footnotetext{
${ }^{1}$ More pictures and videos can be found at URL:www.gel.usherb.ca/laborius/DRF.
} 
robots using directional vision can move in flexible formation, as opposed to rigid formation. Formation switching in our case involves the assignation of a new leader, while in [5] special cases must be programmed to keep robots organized according to their ID numbers. Work by Fierro et al. [3] also uses vision, but with omnidirectional cameras. With such sensors, our approach would not need robots to go into the Rotation-360 state to perceive nearby robots. Formation switching must also preserve ordering of the robots based on their ID, while in our case the switch of formation can be influenced by the situation experienced by the robots. Our approach also demonstrates increased capabilities by being able to address problem d) for establishing a formation. This requires information of global nature concerning the group. Since it cannot be accomplished with robots having limited perception and relative positioning, this is done at the cost of increased communication exchanges between the robots. But our global coordination scheme is robust to local failure of robots in the formation: this would simply lead to a reassignment of the formation.

\section{Conclusion and Future Work}

This paper addresses formation problems of group behavior of multi-robot / distributed systems. Such problems have many dimensions and characteristics, which are difficult to identify in research works conducted on the topic. In an attempt to better understand the challenges of this research topic (which has gained in interest over the past 3 years because of the increased capabilities of mobile robots), the first contribution of this paper is to propose a formalism to characterize formation problems. Based on this categorization and with the long-term objective of doing robot formation control in real life settings, the paper presents a hybrid control approach that allows a group of robots initialize a formation, a capability that had not been addressed in robot formation, in addition to move in formation, avoid obstacles and switch formations. It demonstrates that the approach works with mobile robots using directional visual perception as the primary sense. The paper shows that control of the pan of the camera can increase the adaptability of the formation. Another contribution is that sharing local information between the robots can be exploited by a distributed search algorithm, allowing the group to configure itself at a global level. Future works involve improving the search algorithm to deal with robot that are assigned to another that is not directly visible, and integrating capabilities such as feasibility of formation [12], dynamic switching of formation [2] and merging of formations [8].

\section{Acknowledgments}

F. Michaud holds the Canada Research Chair (CRC) in Mobile Robotics and Autonomous Intelligent Systems. This research is supported financially by CRC, NSERC, CFI and FCAR. The authors also want to thank USC Robotics Research Labs to have made available Player / Stage: Networked Transducer Interface / Multiple Robot Simulator Environment.

\section{References}

[1] T. Balch and R. C. Arkin. Behavior-based formation control for muli-robot teams. IEEE Trans. Robotics and Automation, 14(6):1-15, 1998.

[2] J. D. Desai, V. Kumar, and J. P. Ostrowski. Control of changes in formation for a team of mobile robots. In Proc. IEEE Int. Conf. on Robotics and Automation, pages $1556-1561,1999$.

[3] R. Fierro, A. K. Das ans V. Kumar, and J. P. Ostrowski. Hybrid control of formations of robots. In Proc. Int. Conf. on Robotics and Automation, pages 157-162, 2001.

[4] R. Fierro, P. Song, A. K. Das, and V. Kumar. Cooperative control of robot formations. Submitted to Cooperative Control and Optimization Series. Kluwer, 2001.

[5] J. Freslund and M. J. Mataric. A general, local algorithm for robot formations. Submitted to IEEE Trans. Robotics and Automation, Special Issue on Multi-Robot Systems, 2001.

[6] B. P. Gerkey, R. T. Vaughan, K. Stoy, A. Howard, G. S. Sukhatme, and M. J. Mataric. Most valuable player: A robot device server for distributed control. In Proc. IEEE/RSJ Int. Conf. on Intelligent Robots and Systems, pages 1226-1231, 2001.

[7] J. Lawton, B. Young, and R. Beard. A decentralized approach to elementary formation manuevers. In Proc. IEEE Int. Conf. on Robotics and Automation, 2000.

[8] M. A. Lewis and K.-H. Tan. High precision formation control of mobile robots using virtual structures. Autonomous Robots, 4:387-403, 1997.

[9] F. Michaud, G. Lachiver, and C.T. Le Dinh. Architectural methodology based on intentional configuration of behaviors. Computational Intelligence, 17(1), 2001.

[10] F. E. Schneider, D. Wildermuth, and H.-L. Wolf. Motion coordination in formations of multiple mobile robots using a potential field approach. In Distributed Autonomous Robotic Systems, pages 305314. Springer, 2000.

[11] I. Suzuki and M. Yamashita. Agreement on a common $\mathrm{x}-\mathrm{y}$ coordinate system by a group of mobile robots. In Proc. Dagstuhl Seminar on Modeling and Planning for Sensor-Based Intelligent Robot Systems, 1996.

[12] P. Tabuada, G. J. Pappas, and P. Lima. Feasible formations of multi-agent systems. In Proc. American Control Conference, 2001. 